environment. The importance of this approach is most clearly seen in so-called functional disorders such as habit-spasms or allergic conditions where stressful situations induce exacerbations of urticaria or eczema. The evidence that personality and psychological factors play an important part in the major eye diseases is much less convincing.

The fact that cataract is common in mental defectives does not help in elucidating the cause of senile cataract. No clear-cut evidence exists that glaucoma is associated with a specific type of personality, although emotional factors may precipitate an attack of acute glaucoma in those predisposed to the disease by anatomical factors which themselves are genetically controlled.

Eyestrain is the one ophthalmological condition in which psychological factors are all-important, and it is this chapter of the book which has most to commend it to the ophthalmologist. The symptoms of eyestrain, as the author clearly demonstrates, cannot be easily correlated with organic disease of the eyes or optical anomalies such as refractive errors. "Eyestrain develops because the patient is unable to live fully in the visual world." "The visual world gives a person a sense of stability; therefore it is not surprising that if he ceases to live fully in the visual world, he may feel unstable and sick. Conversely, any condition that makes a person feel unstable may diminish the apparent stability of the visual world." There is undoubtedly a great deal of truth in these explanations and the traditional correction of refractive errors and the prescribing of soothing lotions act largely by encouraging the patient to come to terms with circumstances and are often little more than placebos.

Much has been written previously concerning the psychological reactions to blindness, and the brief chapter in this book serves as a reminder of the difficulties of adjustment experienced by blind persons. It is a pity that the author does not deal with the patient's reaction to eye diseases, which, although not necessarily leading to blindness, can undoubtedly produce marked psychological effects.

The first half of the book deals with man's relationship to the visual world in general-disorders of perception, illusions and hallucinations. Again, the emphasis is on the interaction between the visual world and the observer and shows that neither ean be studied in isolation. The final chapters on treatment reveal the difficulties of treating any psychological disturbance but do not add much of value to the therapeutic armamentarium of the ophthalmologist. The final phenomenological analysis of a patient seems to me almost totally irrelevant to ophthalmological practice.

In summary, this is a courageous attempt to cover an extremely wide field, and though it may fail to convert the average ophthalmologist it should make him aware of the wider horizons beyond the limits of his ophthalmoscope. The book is well produced and the bibliography is extensive.

E. S. PERKINS

\section{AUTOMATED GEOLOGY}

\section{Computer Applications in Stratigraphic Analysis}

By John W. Harbaugh and Daniel F. Marriam. Pp. xii + 282. (Wiley: London, August 1968.) 140s.

THE purpose of the book is to describe and illustrate a selection of the principal computerized methods of analysis of stratigraphic data, with an emphasis on techniques of use in problems involving the determination of spatial relationships of data. The book is intended primarily as a reference text for workers in stratigraphy, sedimentation, structural geology and palaeontology, and to a lesser extent in exploration in the petroleum and mining industries, and for workers in different fields where the methods have application. Users of the text are expected to have a moderate background in geology, a working knowledge of algebra, and some knowledge of elementary statistics, basic appreciation of calculus and matrix algebra would be useful, though knowledge of computer hardware and programming is not assumed. The description of the techniques is designed to cater for non-mathematical geologists with reference only to mathematical aspects where necessary.

Seven principal classes of application are considered-. data storage and retrieval methods and automated information systems; mapping techniques including machine contour mapping; time-trend analysis and related elementary smoothing techniques; various forms of least squares trend surface analysis, including conventional polynomial trend analysis and harmonic trend analysis; classification systems; and the numerous forms of mathematical simulation, particularly those that deal with dynamic systems with numerous, interdependent geological variables.

The text, prepared by two leading authorities in the field, is an extremely well constructed leview and examples are lucidly presented of a wide variety of data handling and interpretational problems largely drawn from special. ized publications. The clarity of presentation should serve to whet the appetite of workers for the benefit to be derived by the use of computers in a broad spectrum of specializations but with no more than a working knowledge of interpretational problems facing stratigraphers. Undoubtedly the techniques described have a much wider application in the Earth sciences than hitherto realized, and this text will serve as an excellent source book.

IAN NICHOI

\section{FIRST COURSE}

\section{A First Course in Functional Analysis}

By Martin Davis. (Notes on Mathematics and Its. Applications.) Pp. xii +110 . (Nelson: London, July 1968.) $50 s$.

THIs volume, like its companions in the series to which it belongs, consists of "reprinted lecture notes, edited at least to a satisfactory level of completeness and intelligibility, though not necessarily to the perfection which is expected of a book". It seoms very questionable as to whether the publishing world should produce a work on such a basis, especially at the high price of fifty shillings. for one hundred and ten pages. The printing is very clear, however, and the exposition, although somewhat condensed, should be of value to the pure mathematician who decides on a purchase.

The author sets out with a rather deep ambition, namely, "to demonstrate the essential unity of twentieth century mathematics", but, of course, it need hardly be said that this is only partly achieved. On the other hand, the "unity" of algebra and analysis is demonstrated by careful preparation for, and expounding of, the proof by Gelfand, via maximal ideals, of Wiener's theorem that if an absolutely convergent trigonometric series has a nonvanishing sum, the reciprocal of the sum can also be expanded into a similar series. The steps proceed via chapters on normed linear spaces and algebras, functions. on Banach spaces and homomorphisms on normed linear spaces.

The book arises out of a lecture course given in 1959 to. graduate students "with quite modest preparation". Each chapter is accompanied by a set of problems which were previously assigned to the students on the course. It is surprising that there is no bibliography and the student who wishes to read further will have to look for references in other texts on the subject.

L. S. GODDARD 